Supporting Information

\title{
Drop Mixing in a microchannel for lab-on-a-chip platforms
}

\author{
Minsoung Rhee ${ }^{1}$ and Mark A. Burns ${ }^{1,2, *}$ \\ ${ }^{1}$ Department of Chemical Engineering, the University of Michigan \\ 2300 Hayward St. 3074 H.H. Dow Building, Ann Arbor, MI 48109-2136 \\ ${ }^{2}$ Department of Biomedical Engineering, the University of Michigan
}

2200 Bonisteel Blvd, Ann Arbor, MI 48109-2099

AUTHOR EMAIL ADDRESS:

Minsoung Rhee (minsoung@umich.edu)

Mark A. Burns* (maburns@umich.edu) 


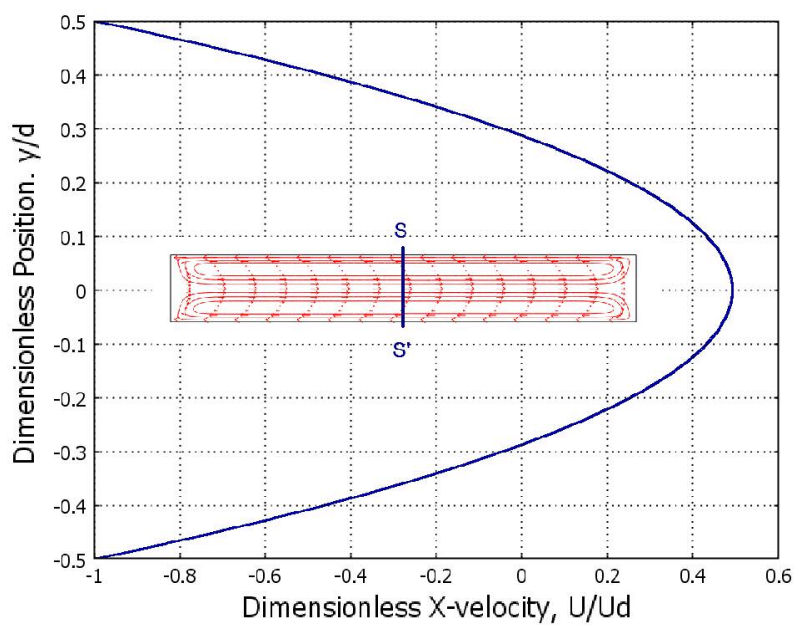

(c)

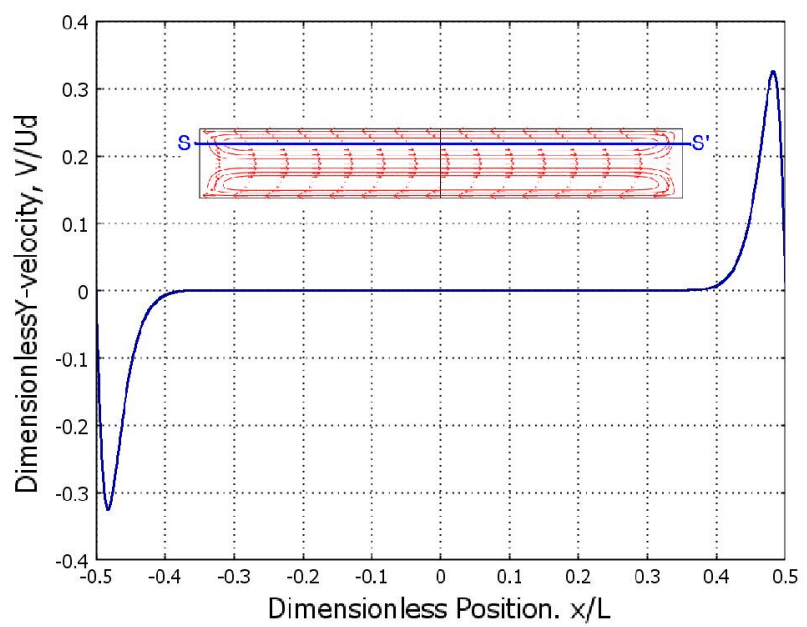

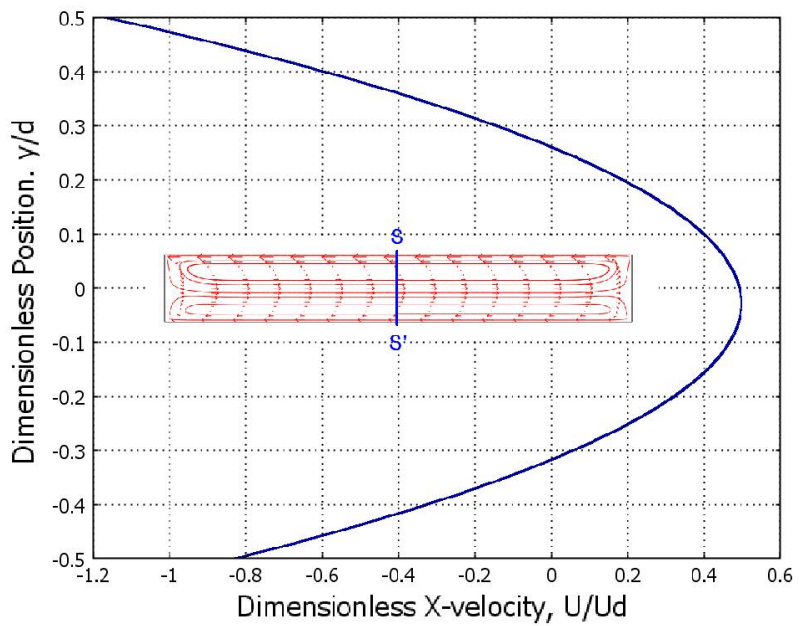

(d)

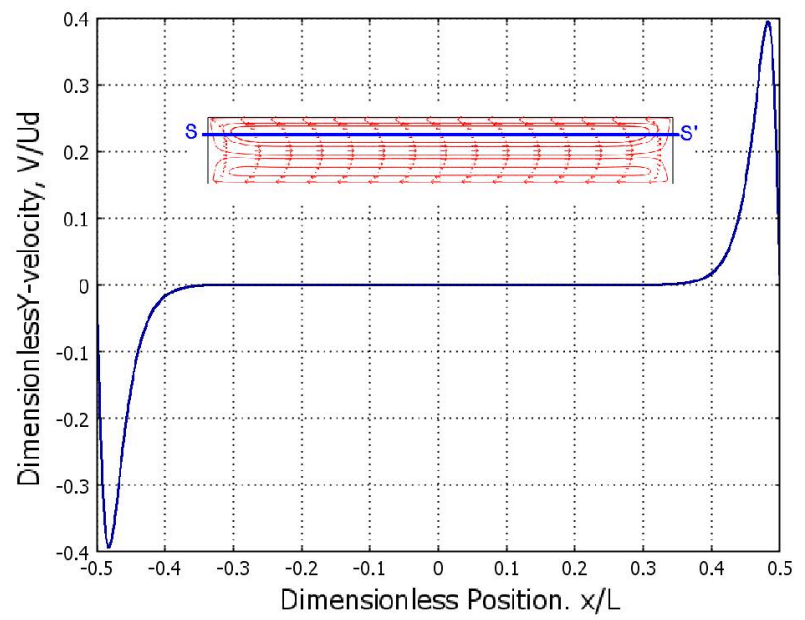

Figure $S 1$. Velocity profiles for a drop of $\varepsilon=7$. (a) $x$-velocity (at $x=0, S^{\prime}$ ) in the straight channel. (b) $x$ velocity (at $\mathrm{x}=0$ ) in the curved channel $(\mathrm{w}=1 \mathrm{~mm}$ and the radius of curvature $=4.2 \mathrm{~mm})$. (c) $\mathrm{y}$-velocity (at $y / d=0.29$ ) in the straight channel. (d) $y$-velocity (at $y / d=0.29$ ) in the curved channel. An approximately $17 \%$ increase from the straight-channel velocity at the outer wall and a $19 \%$ decrease at the inner wall result from the curved flow. 


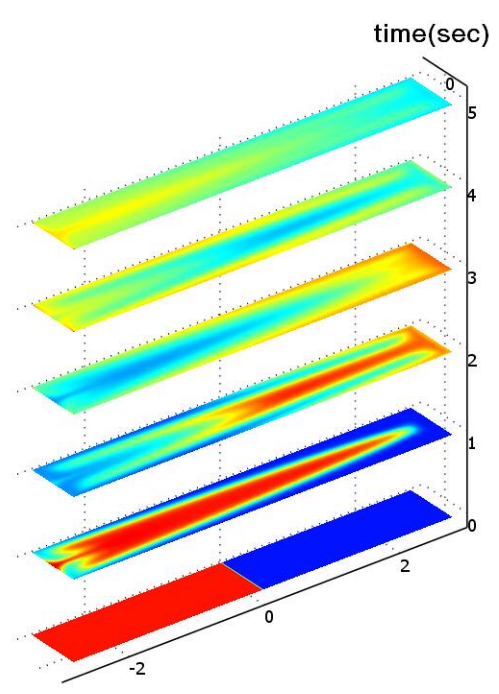

Concentration $\left(\mathrm{mol} / \mathrm{m}^{3}\right)$

(b)
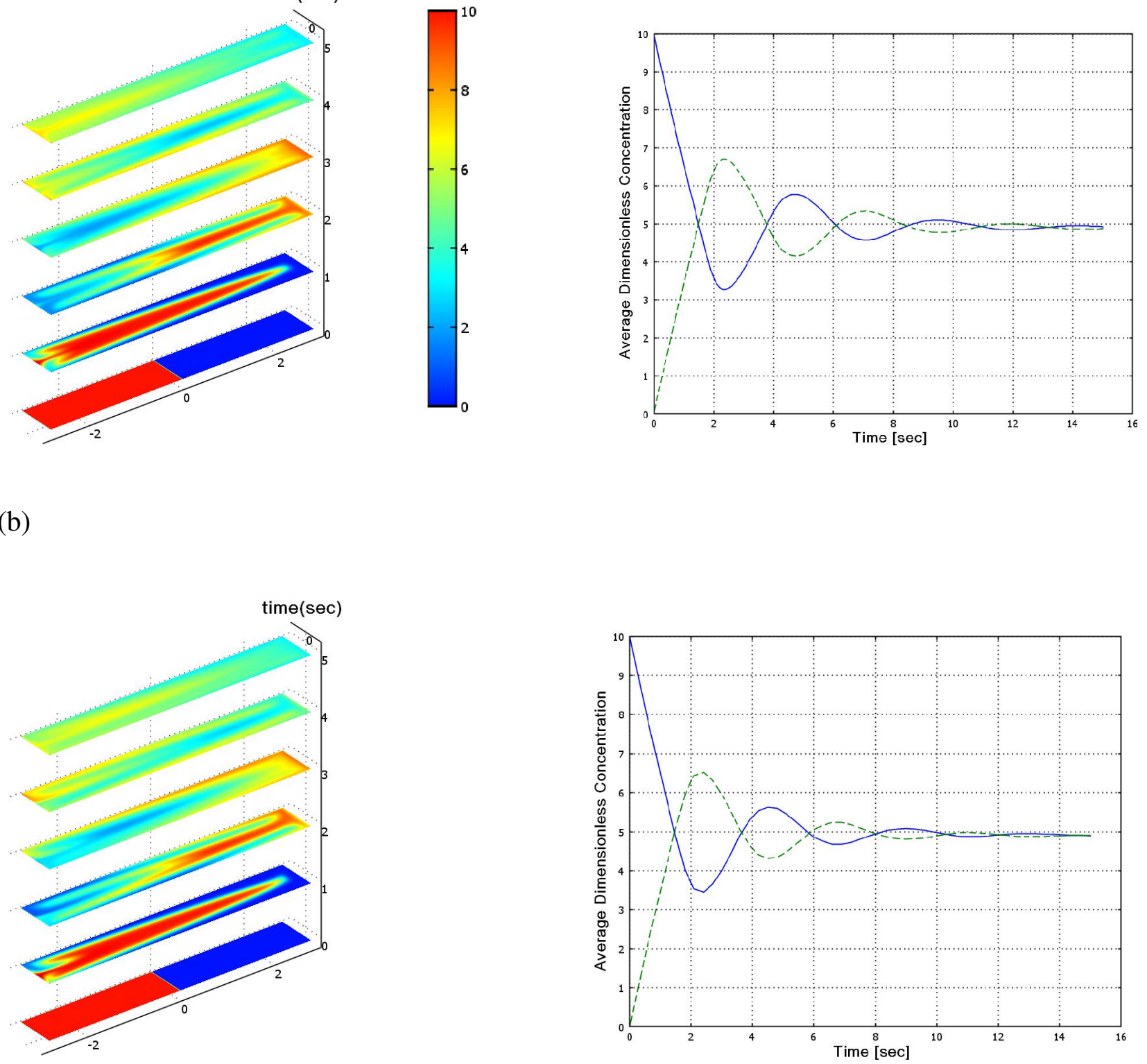

Figure S2. Simulation results of convection-dominated mixing in a (a) straight and (b) curved channel $(\mathrm{w}=1 \mathrm{~mm}$ and the radius of curvature $=4.2 \mathrm{~mm})$ for $\mathrm{Pe}^{*}=100$ and $\varepsilon=7$. The left-side diagrams represent changes of the solute concentration in time as shown in Figure 3. The right-side plots show how the average concentration of the left and right domains changes during the mixing process as seen in Figure 5. Comparing (a) and (b), there is little noticeable difference in mixing time between the straight and curved mixing channels even at the high $\mathrm{Pe}^{*}$. 


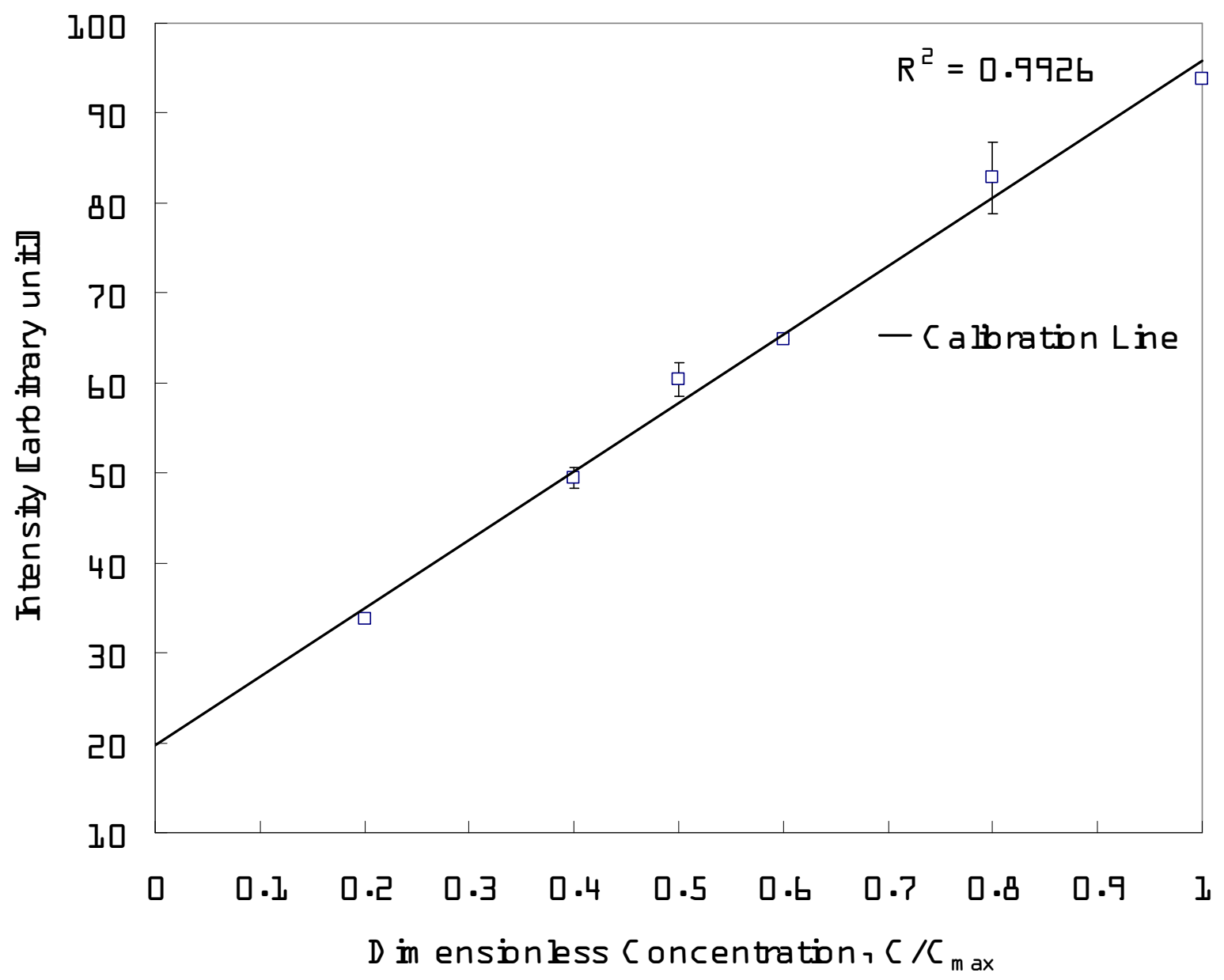

Figure S3. A calibration curve for the luminance intensity and the dye concentration. A blue dye (Trypan blue $0.4 \%$ solution, Sigma-Aldrich) and a colorless liquid (DI water) were used. The images recorded and processed in computer can express 256 levels of the luminance. From the fitting, the concentration of trypan blue dye was considered linearly proportional to the intensity of the recorded image. 